\title{
The place of Dirac's Equation in Five-Dimensional Riemannian Geometry
}

\author{
By H. W. HASKEY.
}

(Received 25th June, 1945. Read 2nd November, 1945.)

$$
\S(1) \text {. }
$$

After a summary of the results of previous writers $\S(2)$, it is shown in $\S(3)$ that if Dirac's equation holds, then $\operatorname{div} D$ vanishes, where $D$ is Whittaker's Euclidean vector. ${ }^{1} D$ may be written in the form $\psi^{\prime} F \psi$, the product of three matrices. In five-dimensional Riemannian geometry Flint's form of Dirac's equation may be rewritten as $\psi^{*} \gamma^{\mu} \frac{\partial \psi}{\partial x^{\mu}}=0$. These modifications of the forms of Whittaker and Flint are later linked thrgugh the geometry of Distant Parallelism, ${ }^{3}$ necessary results in which are given in $\$(4)$. The postulates of $\S(5)$ show the relativistic manner of linkage and the broadening of the Riemannian geometry proposed for this purpose. In $\S(6)$ the effect of this broadening on metrical quantities is examined while in $\S(7)$ the suggested quantum equation is seen to be Dirac's. The dependence of the Dirac $\psi$ 's on space-structure and the interpretation of the coordinate transformations of $x^{5}$ are given in $\S \S(8$ and a) mspectively.

§(2). Summary of known results used later.

With a slight change of notation, Whittaker's vector $D$ has components

$$
\begin{array}{ll}
D^{1}=\psi_{1} \psi_{4}+\psi_{3} \psi_{2}, \quad D^{2}=\imath\left(\psi_{1} \psi_{4}-\psi_{3} \psi_{2}\right), & D^{3}=\psi_{1} \psi_{3}-\psi_{2} \psi_{4}, \\
& D^{4}=\iota\left(\psi_{1} \psi_{3}+\psi_{2} \psi_{4}\right)
\end{array}
$$

1 E. T. Whittaker, Proc. Royal Soc. (A) (158) (1937), 38-46.

2 H. T. Flint, Phil. Mag., 7(29) (1940), $417-433$ (429).

H. T. Flint, Proc. Royal Soc. (A) (150) (1935), 421-441.

3 A. Einstein, Berliner Sitzungberichte (1928), 217-221, 224-227.

A. Einstein, Berliner sitzungberichte (1929), 2-7, 156.159.

A. Einstein, Berliner Sitzungberichte (1930), 18-23, 401-402.

A. Einstein. W. Mayer, Berliner Sitzungberichte (1931), 257-265.

R. Weitzenbock, Rerliner Sitzungberichte (1928), 466.474.

H. T. Flint, Proc. Royal Soc. (A) (121) (1928), 676-681. 
where $\left(\psi_{1}, \psi_{2}\right)$ and $\left(\psi_{3}, \psi_{4}\right)$ are spinors transforming according to $\psi_{1}^{\prime}=a \psi_{1}+\beta \psi_{2} ; \quad \psi_{2}^{\prime}=\gamma \psi_{1}+\delta \psi_{2} \quad(2 a) \quad$ where $a \delta-\beta \gamma=1$, the metric being given by $d s^{2}=\left(d x^{1}\right)^{2}+\left(d x^{2}\right)^{2}+\left(d x^{3}\right)^{2}+\left(d x^{4}\right)^{2}$. The vector $D$ is self-perpendicular so that

$$
\left(D^{1}\right)^{2}+\left(D^{2}\right)^{2}+\left(D^{9}\right)^{2}+\left(D^{4}\right)^{2}=0
$$

The Riemannian interval $d \sigma$ is given by

$$
d \sigma^{2}=\gamma_{\mu \nu} d x^{\mu} d x^{\nu} \quad(5 \mathrm{a}) \quad \text { where } \mu, \nu=1,2,3,4,5 .
$$

Greek scripts relate to a five-dimensional world. Kaluza and Klein ${ }^{2}$ take this to be cylindrical. Hence

$$
\gamma_{\mu \nu} \text { is independent of } x^{5} \text {. }
$$

The three-index symbol is written as $\Gamma_{\beta \gamma}^{a}\left(=\Gamma_{\gamma \beta}^{a}\right)$. The Tetrode matrices, $\gamma^{\mu}$, are connected with the $\gamma^{\mu \nu}$ by the equations

$$
\gamma^{\mu} \gamma^{\nu}+\gamma^{\nu} \gamma^{\mu}=2 \gamma^{\mu \nu} 1 \text {. }
$$

The covariant derivative of a tensor $T_{\beta}^{a}$ with respect to $x^{\lambda}$ is denoted by $T_{\beta: \lambda}^{a}$. Schroedinger ${ }^{4}$ writes

$$
\gamma_{\mathrm{t}: \lambda}=\Gamma_{\lambda} \gamma_{\mathrm{t}}-\gamma_{\mathrm{c}} \Gamma_{\lambda}
$$

The permissible transformations of coordinates in this cyludrical world are governed by

$$
\begin{aligned}
& x^{5}=\bar{x}^{5}+f_{5}\left(\bar{x}^{1}, \bar{x}^{2}, \bar{x}^{3}, \bar{x}^{4}\right) \\
& x^{m}=f_{m}\left(\bar{x}^{1}, \bar{x}^{2}, \bar{x}^{3}, \bar{x}^{4}\right) .
\end{aligned}
$$

To include electromagnetism with gravitation in this world, Klein takes

$$
\begin{aligned}
& \gamma_{m 5}=k \omega \phi_{m}, \quad \gamma_{m n}-g_{m n}=\left(\gamma_{5 m} \cdot \gamma_{5 n}\right) / \omega^{2} \\
& \gamma^{m 5}=-\left(k \phi^{m}\right) / \omega, \quad \gamma^{55}=\left(k^{2} \phi^{m} \phi_{m}+1\right) / \omega^{2},
\end{aligned}
$$

where $m, n=1,2,3,4, k$ is a constant connecting gravitational and electromagnetic units, $g_{m n}$ is the metrical tensor of General Relativity corresponding to $\gamma_{\mu \nu}$ and the constant $\omega$ is given by

Further, the expressions

$$
\omega^{2}=\gamma_{55}
$$

$$
\left.d \theta=\left(\gamma_{\mu 5} d x^{\mu}\right) / \omega \quad(13 a) \quad r_{r n n} \quad v_{n m o} \gamma_{n 5}\right) / \omega^{2}
$$

1 Th. Kaluza, Berliner Sitzungberichte (1921), 966-972.

2 O. Klein, Zeischrift für Phys. (37) (1926), 895-906.

${ }^{3}$ H. Tetrode, Zeitschrift für Phys. (50) (1928), 336-346.

4 E. Schroedinger, Berliner Sitzungberichte (11) (1932), 105-128 (109). 
and

$$
\frac{\partial}{\partial x^{n}}\left(\frac{\gamma_{m 5}}{\omega}\right)-\frac{\delta}{\partial x^{m}}\left(\frac{\gamma_{n 5}}{\omega}\right)
$$

are invariant under $(9 a)$ and $(10 a)$.

The immediate vicinity of every point of a Riemannian continuum is the seat of the embedded world of Einstein's Distant Parallelism, determined by tensors $h_{a}^{\mu}, h_{a \mu}$, in which components relating to the embedded geometry are denoted by the first subscrip after the $h$, while Riemannian components are indicated by the ensuing super-or subscript.

We have

$$
\begin{aligned}
& \gamma_{\mu \nu}=h_{a \mu} \cdot h_{a \nu} \\
& \gamma^{\mu \nu}=h_{a}^{\mu} \cdot h_{a}^{\nu} \\
& L^{\mu}=h_{a}^{\mu} L_{a}
\end{aligned}
$$

where $L^{\mu}$ and $L_{a}$ are corresponding components of the same vector in the two geometries. The three-index symbol appropriate to Distant Parallelism is denated by $\Delta_{\nu \lambda}^{\mu}$ and

$$
\Delta_{\nu \lambda}^{\mu}=h_{a}^{\mu} \frac{\partial h_{a \nu}}{\partial x^{\lambda}}
$$

while the covariant derivative of $T_{\beta}^{a}$ with respect to $x^{\lambda}$ in this genmetry is written as $T_{B ; \lambda^{\circ}}^{a}$. It follows that

$$
h_{a \beta ; \lambda}=0 \text { and } h_{a ; \lambda}^{\beta}=0 \text {. }
$$

The quantity $\Delta_{\alpha \beta}^{\mu}-\Delta_{\beta \alpha}^{\mu}$ is a tensor which $v$ anishes if the continuum is Euclidean everywhere and we write

$$
\Lambda_{\alpha \beta}^{\mu}=\Delta_{\alpha \beta}^{\mu}-\Delta_{\beta \alpha}^{\mu} \quad \text { (2la) } \quad \text { and } \quad \Lambda_{\alpha \mu}^{\mu}=\Lambda_{\alpha} .
$$

The connection between $\Gamma_{\iota \lambda}^{\mu}$ and $\Delta_{\iota \lambda}^{\mu}$ is given by

$$
2 \Gamma_{\iota \lambda}^{\mu}=\Delta_{\iota \lambda}^{\mu}+\Delta_{\lambda_{\iota}}^{\mu}+\gamma^{\mu a} \gamma_{\iota \beta} \Lambda_{a \lambda}^{\beta}+\gamma_{\lambda \beta} \gamma^{\mu a} \Lambda_{a, ~}^{\beta}
$$

and the simplest connection between $\gamma^{\mu}$ and $h_{a}^{\mu}$ is given by

$$
\gamma^{\mu}=h_{a}^{\mu} E_{a}, a=1,2,3,4,5
$$

where $E_{a}$ are five Dirac matrices forming a pentad of which the one used later is

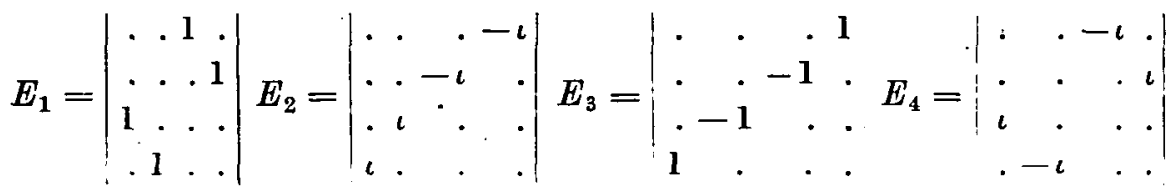

$$
\begin{aligned}
& E_{5}=\left|\begin{array}{rrrr}
1 & \cdot & \cdot & \cdot \\
\cdot & 1 & \cdot & \cdot \\
\cdot & \cdot & -1 & \cdot \\
\cdot & \cdot & \cdot & -1
\end{array}\right|
\end{aligned}
$$

where a dot indicates a zero element. Tue $E$ 's are Hermitean. 
If $\psi=\left(\psi_{1}, \psi_{2}, \psi_{3}, \psi_{4}\right)$ is a row vector in cylindrical five-dimensional geometry in which $\omega$ is a constant, Flint obtains Dirac's equation in the form

$$
\gamma^{\mu} \frac{\partial \psi}{\partial x^{\mu}}=0 \quad(26 a) \quad \text { where } \quad \frac{\partial \psi}{\partial x^{5}}=\frac{2 \pi \iota m_{0} c}{h} \psi
$$

and $\psi$ is a scale-factor after the manner of Weyl.

$$
\S(3) \text {. }
$$

From now on new equations are numbered (1), (2), etc., the known results of $\S(2)$ are quoted as (1a), (2a), etc.

From (la) the equation div $D=0$ becomes

$$
\begin{aligned}
& \psi_{3}\left(\iota \frac{\partial \psi_{1}}{\partial x^{4}}+\frac{\partial \psi_{2}}{\partial x^{1}}-\imath \frac{\partial \psi_{2}}{\partial x^{2}}+\frac{\partial \psi_{1}}{\partial x^{3}}-b \psi_{4}\right) \\
+ & \psi_{4}\left(\iota \frac{\partial \psi_{2}}{\partial x^{4}}+\frac{\partial \psi_{1}}{\partial x^{1}}+\iota \frac{\partial \psi_{1}}{\partial x^{2}}-\frac{\partial \psi_{2}}{\partial x^{3}}+b \psi_{3}\right) \\
+ & \psi_{1}\left(\iota \frac{\partial \psi_{3}}{\partial x^{4}}+\frac{\partial \psi_{4}}{\partial x^{1}}+\iota \frac{\partial \psi_{4}}{\partial x^{2}}+\frac{\partial \psi_{3}}{\partial x^{3}}+b \psi_{2}\right)=0, \\
+ & \psi_{2}\left(\iota \frac{\partial \psi_{4}}{\partial x^{4}}+\frac{\partial \psi_{3}}{\partial x^{1}}-\imath \iota \frac{\partial \psi_{3}}{\partial x^{2}}-\frac{\partial \psi_{4}}{\partial x^{3}}-b \psi_{1}\right)
\end{aligned}
$$

where $b h=2 \pi \iota m_{0} c$.

In (1) certain terms containing $b$ have been added but the sum of such terms is zero and the equation takes the form

where

$$
\psi_{r} \Omega_{r}=0 \quad r=1,2,3,4,
$$

are Dirac's equations. Hence (1) holds when Dirac's equations hold.

Since the coefficients of the $c \psi / \partial x^{m}$ and of $b \psi$ in (3) are elements of $E$-matrices, the vector $D$ may be written as

$$
\begin{aligned}
& 2 D^{m}=\psi^{\prime} F^{m} \psi \quad m=1,2,3,4 \\
& 2 D^{5}=\psi^{\prime} F^{5} \psi=0,
\end{aligned}
$$

where the $F$ 's form a set of five four-point matrices, and $\psi^{\prime}$ is the transposed of $\psi$.

The vector character of $D$ has thus been made dependent on the vector character of the four-point matrices $F$, the $\psi$ 's being regarded as invariants. This procedure seems justified by the remarks of Laporte and Uhlenbeck ${ }^{1}$ who give alternatively the $F^{\prime}$ 's as constants and the $\psi$ 's transformables.

\footnotetext{
${ }^{1}$ Laporte and Uhlenbeck, Phys. Rev. 2nd Series (37) (2) (1931), 1380-1397.
} 
Flint's equation (26a) may be written as

$$
\psi^{*} \gamma^{\mu} \frac{\partial \psi}{\partial x^{\mu}}=0
$$

where $\psi^{*}$ is the complex conjugate of $\psi$, and be linked with Whittaker's work by passing from Euclidean to Riemannian Geometry via Distant Parallelism.

$\S(4)$. Some properties of Distant Parallelism.

From (21a), (2£a) it follows that

$$
\Lambda_{\mu}=h_{a}^{a}\left(h_{a \mu ; a}-h_{a a: \mu}\right)
$$

and, by differentiating the equation $h_{a}^{a} \cdot h_{a a}^{\cdot}=\delta_{a}^{\alpha}$ with manipulation of dummy suffixes, we have

which, with (6), gives

$$
h_{a a} \cdot h_{a: \mu}^{a}=0
$$

$$
\begin{aligned}
& \Lambda_{\mu}=h_{a}^{a} \cdot h_{a \mu ; a} \\
& \Lambda_{\mu}=-h_{a \mu} \cdot h_{a: a}^{a} \\
& h_{a}^{\mu} \Lambda_{\mu}=-h_{a: \mu}^{\mu} .
\end{aligned}
$$

Using (25a), (20a) and (24a) and Laporte's second alternative $\S(3)$ it follows that

Also (24a) yields

$$
\gamma_{: a}^{\mu}=0
$$

$$
2 h_{a}^{\mu}=\operatorname{trace}\left(\gamma^{\mu} E_{a}+E_{a} \gamma^{\mu}\right)
$$

so that

$$
2 h_{a: \mu}^{\mu}=\operatorname{trace}\left(\gamma_{: \mu}^{\mu} E_{a}+E_{a} \gamma_{: \mu}^{\mu}\right) \text {. }
$$

Hence by (9), $\quad 2 \Lambda_{a}=-\operatorname{trace}\left(\gamma_{: \mu}^{\mu} \gamma_{a}+\gamma_{a} \gamma_{: \mu}^{\mu}\right)$

and, by (10) and (24a),

It is found that

$$
\gamma_{: a}^{a}=-\gamma^{a} \Lambda_{a}
$$

$$
2 \gamma_{\iota: \lambda}=\left(\gamma_{\lambda a} \Lambda_{\iota \mu}^{a}+\gamma_{\iota a} \Lambda_{\lambda_{\mu}}^{a}+\gamma_{\mu a} \Lambda_{\iota \lambda}^{a}\right) \gamma^{\mu}
$$

by use of (23a) and (24a), and this leads to the value of $\Gamma_{\lambda}$ in (8a) namely

$$
-8 \Gamma_{\lambda}=\left(\gamma_{\lambda a} \Lambda_{\mu \nu}^{a}+\gamma_{\mu a} \Lambda_{\lambda \nu}^{a}-\gamma_{\nu a} \Lambda_{\lambda \mu}^{a}\right) s^{\mu v}+t_{\lambda} \cdot 1
$$

where $2 s^{\mu \nu}=\gamma^{\mu} \gamma^{\nu}-\gamma^{\nu} \gamma^{\mu}$ and $t_{\lambda}$ is an arbitrary vector.

1 H. T. Flint, Phil. Mag. (7) (29) (1940), $417-433$ (429). 


\section{$\S(5)$. Postulates.}

A. The vector $\psi^{*} \gamma^{\mu} \psi$, where the four non-vanishing components of $\psi^{*}$ and $\psi$ are invariants in a cylindrical Riemannian five-world, is the generalised Whittaker vector. $\psi^{*}$ is the complex conjugate of $\psi$.

B. The generalised Whittaker vector is identified with the basic vector of Distant Parallelism by the equation

$$
\Lambda^{\mu}=\psi^{*} \gamma^{\mu} \psi
$$

C. The fundamental law of atomic mechanics is given by

$$
\Lambda_{: \mu}^{\mu}=0
$$

in a cylindrical world more general in character than that of Klein.

D. The generalisation in $\mathrm{C}$ is obtained by setting

$$
\omega^{2}=\gamma_{55}=F^{\prime}\left(x^{1}, x^{2}, x^{3}, x^{4}\right) \text {. }
$$

E. When atomic phenomens are considered $\omega$ is given by $\mathrm{D}$ and gravitation is neglected so that $g_{m n}=1$ or 0 according as $m, n$ are the same or different.

F. The dependence of $\psi$ in $A$ on $x^{5}$ is given by

$$
\frac{\partial \psi}{\partial x^{5}}=\frac{2 \pi \iota m_{0} c \omega}{h} \psi
$$

G. The constant $k$ in (lla) loses its meaning when gravitation is neglected, in which case we set

$$
e / k=m_{0} c^{2}
$$

$$
\S(6) \text {. }
$$

Postulates A, B, C are suggested by $\S(3)$. The generalisation D does not affect the invariance of (12a), (13a) and (14a) under transformations (9a) and (10a), but the expression (15a), still to be regarded as the electromagnetic intensity, will be invariant only if

$$
\frac{\partial f_{5}}{\partial x^{m}} \cdot \frac{\partial \omega}{\partial x^{n}}=\frac{\partial f_{5}}{\partial x^{n}} \cdot \frac{\partial \omega}{\partial x^{m}},
$$

that is, if $\omega$ and $f_{5}$ are functions of each other. We then have $\omega$ invariant and $(9 a)$ becomes

$$
x^{5}=\bar{x}^{5}+f(\omega)
$$


Using $\mathrm{E}$, it is found that one $\mathrm{se}^{\prime} \cdot{ }^{\prime} \cdot h_{a}^{\mu}$ which satisfy (11a) is

$$
\begin{aligned}
& h_{11}=h_{22}=h_{33}=h_{44}=1=h_{1}^{1}=h_{2}^{2}=h_{3}^{3}=h_{4}^{4} \\
& h_{5 m}=k \phi_{m}, h_{55}=\omega, h_{m}^{5}=-\left(k \phi^{m}\right) / \omega, h_{b}^{5}=1 / \omega
\end{aligned}
$$

where $m=1,2,3,4$ and $k$ is given in G. With these values the only components of $\Lambda_{\mu \nu}^{a}$ which do not vanish are

$$
\begin{aligned}
& \Lambda_{m n}^{\delta}=\left(k F_{m n}\right) / \omega \\
& \Lambda_{m b}^{\delta}=\Lambda_{m}=\Lambda^{m}=-\frac{\partial \log \omega}{\partial x^{m}},
\end{aligned}
$$

in which $F_{m n}=\frac{\partial \phi_{m}}{\partial x^{n}}-\frac{\partial \phi_{n}}{\partial x^{m}}$ is the electromagnetic intensity.

With (24a), (23) gives

$$
\gamma^{m}=E^{m} \text { and } \gamma^{5} \omega=-k \phi^{m} E^{m}+E^{5} .
$$

$\S(7)$. The suggested Quantum Equation.

From (18), (19), (11) we have

$$
\frac{\partial \psi^{*}}{\partial x^{\mu}} \gamma^{\mu} \psi+\psi^{*} \gamma^{\mu} \frac{\partial \psi}{\partial x^{\mu}}=\mathbf{0}
$$

which, with (26), (21) and (20) yields

$$
\begin{aligned}
\left(H \frac{\partial \psi^{*}}{\partial x^{m}} E^{m}+\right. & \left.\frac{e}{c} \phi^{m} \psi^{*} E^{m}-m_{0} c \psi^{*} E^{5}\right) \psi \\
& +\psi^{*}\left(H E^{m} \frac{\partial \psi}{\partial x^{m}}-\frac{e}{c} \phi^{m} E^{m} \psi+m_{0} c E^{5} \psi\right)=0,
\end{aligned}
$$

where $2 \pi \iota H=h$. Writing $\Omega=H E^{m} \frac{\partial \psi}{\partial x^{m}}-\frac{e}{c} \phi^{m} E^{m} \psi+m_{0} c E^{5} \psi$, and remembering that $E^{m}, E^{5}$ are Hermitean, (28) assumes the form

$$
\text { trace }\left(\Omega^{*} \psi+\psi^{*} \Omega\right)=0 \text {, }
$$

in which $\Omega^{*}$ denotes the complex conjugate of $\Omega$. Setting $\Omega_{q}=R_{q} \exp \left(\iota \theta_{q}\right)$ and $\psi_{q}=r_{q} \exp \left(\iota a_{q}\right),(29)$ becomes $R_{q} r_{q} \cos \left(\theta_{q}-a_{q}\right)=0$.

Since, in general, $\theta_{q}-a_{q}$ differs from $\left(2 n+\frac{1}{2}\right) \pi$ and, by $A, r_{q}$ differs from zero, it is concluded that $R_{q}$ vanishes and that

$$
\Omega_{q}=0 \text {. }
$$


It then appears that the $\psi$ 's in (30) are Dirac Wave-functions and that the equation

$$
\Lambda_{; \mu}^{\mu}=0
$$

is Dirac's equation when $B$ is used.

The step from classical to quantum mechanics involves (1) the promotion of $\omega$ from a constant to an invariant and (2) the transition from the Riemannian continuum to that of the embedded world of Distant Parallelism. The relationship between the Dirac $\psi$ 's and the metrical tensor $\Lambda_{\mu}$ is, by (18) and (25a),

$$
\Lambda_{1}=\psi_{1}^{*} \psi_{3}+\psi_{2}^{*} \psi_{4}+\psi_{3}^{*} \psi_{1}+\psi_{4}^{*} \psi_{2}
$$

and four similar components, the last of which is zero. From these it follows that

$$
\Lambda^{\mu} \Lambda_{\mu}=\left[\operatorname{trace} \psi^{*} \psi\right]^{2}
$$

so that the length of $\Lambda_{\mu}$ is proportional to the probability of occurrence of the electron.

From (15), (25), (26) we have

$$
\gamma_{: \mu}^{\mu}=E^{m} \frac{\partial \log \omega}{\partial x^{m}} \quad \text { (32) } \quad \text { and trace } \gamma_{: \mu}^{\mu}=0 \text {. }
$$

Using (25a) with (32) it is found that

$$
2\left(\psi_{2}^{*} \psi_{4}+\psi_{3}^{*} \psi_{1}\right)=-\left(\gamma_{: \mu}^{\mu}\right)_{13}=\Lambda^{1}+\iota \Lambda^{4}=-\left(\gamma_{: \mu}^{\mu}\right)_{42}=-\left(\frac{\partial}{\partial x^{1}}+\iota \frac{\partial}{\partial x^{4}}\right) \log \omega
$$

and three other similar results. Thus, apart from their dependence on $x^{5}$, the Dirac $\psi$ 's are more truly part of the geometric stage than actors on it and this is a sine qua non of a relativistic theory.

\section{$\S(9)$. The Coordinate Transformation of $x^{5}$.}

The unidentified fifth coordinate, $x^{5}$, transforming according to (22), behaves differently from the others. It is a cyclic coordinate and its rôle, to use Whittaker's metaphor, is that of a catalytic agent. By postulate A the $\psi$ 's are Riemannian invariants and will be unchanged in value by the transformation (22) which yields

so that (22) becomes

$$
m_{0} c \omega f(\omega)=n h,
$$

$$
x^{5}=\bar{x}^{5}+n h /\left(m_{0} c \omega\right)
$$


where $n$ is an integer. Hence at any point, $P$, of the continuum $x^{5}$ can be changed only by an integral multiple of $h /\left(m_{0} c \omega\right)$, that is, of an invariant at $P$. This restriction, and the freedom to ascribe a meaning to $x^{5}$, suggests that the permissible change in it is a measure of the uncertainty of position of the electron and that $m_{0} c \omega$ is associated with the uncertainty of the (conjugate) momentum.

If $\alpha$ is the angle between the vectors with components $\Lambda_{\mu}$ and $d x^{\mu}$ we have, by (31),

$$
\psi_{m}^{*} \psi_{m} \cdot \cos \alpha \cdot d \sigma=\Lambda_{\mu} d x^{\mu}
$$

so that, by (25),

$$
\omega=\omega_{0} \exp \left(-\int \psi_{m}^{*} \psi_{m} \cos a \cdot d \sigma\right)
$$

$$
\S(10) \text {. Conclusion. }
$$

It must be confessed that in the foregoing a heavy burden has been placed on the invariant $\omega$, that the interpretation of $(36)$ is speculative and that perhaps the $\psi$ 's have been overdetermined by equations (34) and (30).

It has been said that some of the entities appearing in quantum theory have "slipped through the tensor net.". Prof. Whittaker's paper on the relations between the spinor and tensor calculus shows how this has happened and has introduced the important vector $D$ on which this paper is founded.

\section{Summary.}

The generalised Whittaker vector is $\Lambda_{\mu}$ which is prevented from vanishing by rejection of the constancy of $\omega$, previously assumed by all writers. It is shown that (1) the null divergence of $\Lambda^{\mu}$ is equivalent to Dirac's equation, (2) the length of $\Lambda^{\mu}$ measures the probability of occurrence of the electron (3) components of $\Lambda^{\mu}$ are connected with the Dirac wave functions and possible transformations of $x^{6}$ are probably related to the Uncertainty Principle. 